\title{
Creative Disruption in Fintech from Sri Lanka
}

BY AJIT SAMARANAYAKE, SAMPATH TILAKUMARA, THAYAPARAN SRIPAVAN, AND RASIKA WITHANAWASAM

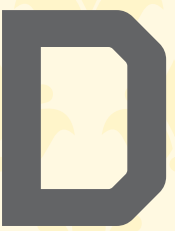

URING THE 1990S, the Sri Lankan IT sector was sandwiched between

the forces of free market competition and the internal turbulence due to civil unrest. The relatively small internal marketplace made it difficult to attract foreign investments and expand businesses beyond IT offshoring. However, stock trading was a brickand-mortar business that presented promising growth potential with the advent of financial technology (fintech).

Sensing this opportunity, a set of seasoned managers at an existing IT services business set off with a broader vision, marking the birth of MillenniumIT (now known as LSEG Technology), a fintech product company. The farsighted entry into fintech, and the experience in mobilizing local talent, contributed to the early success of MillenniumIT.

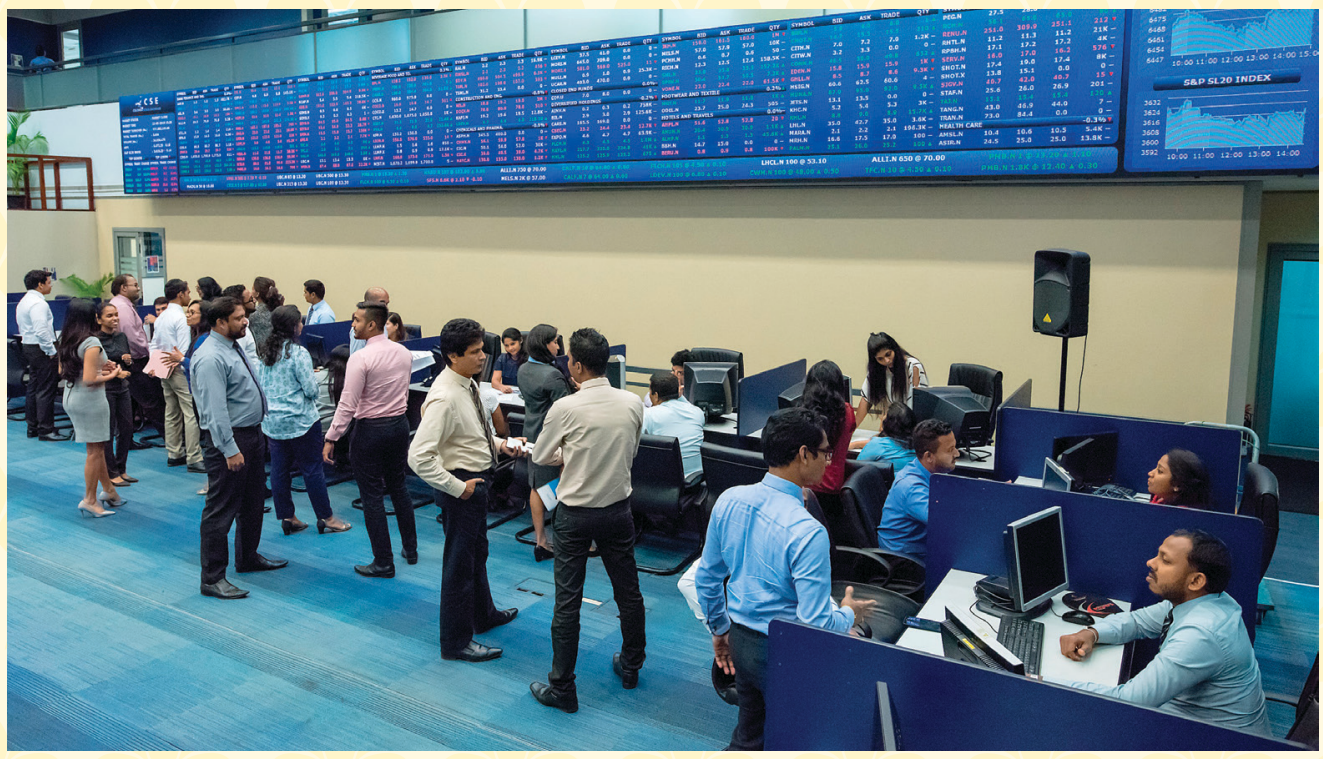

Figure 1. The Colombo Stock Exchange (CSE), the first customer. (Photo courtesy of CSE)

MillenniumIT introduced novel concepts in designing complex electronic trading systems with predictable performance that met the regulated ultra-high resiliency requirements. Being an early mover allowed for the slow, steady penetration of the company's technology into capital markets around the world. In 2009, the London

The early application of "creative disruption' to a niche market with immense growth potential has proven to be a very effective tool and strategy.

\section{Software resilience for scalable distributed systems.}

Scalable distributed systems are at the core of the architecture of the organization's electronic trading systems. Competitive chal- lenges served to help contain the costs of scalability, while preserving high resiliency. LSEG Technology quit using costlier hardware-dependent resiliency by introducing software fault-tolerance models into a freshly built common technology framework for fintech applications.

Such software fault tolerance introduced patented models of replication, synchronization, and recovery that ran on commodity hardware at a fraction of the cost, establishing new industry benchmarks on system availability for mission-critical fintech applications.

A first-of-its-kind deployment in Sri Lanka's national stock exchange (CSE) in 1995 was followed by implementations in global trad- 
ing hubs in London, Milan, Oslo, and Johannesburg.

\section{2. and 3.}

High-performance and heterogeneous computing.

Execution of complex func-

tionality at ultra-low latency

is imperative for electronic

trading systems.

That pattern of confounding expectations led to a number of inflection points when LSEG Technology offered an ultra-lowlatency trading system to address the London Stock Exchange's requirements in 2011. The low-latency external interfaces developed as part of this solution allowed co-located high-frequency traders to take advantage of the ultra-low latency of the platform. A sub $100 \mu$ s step- jump in end-to-end latency was possible with a full-stack re-architecture, and being the first to infuse emerging transport technologies (such as Infiniband) helped the London Stock Exchange gain market share and stay ahead of other leading exchanges.

In 2014, an award-winning ${ }^{8,9}$ low-latency market data distribution platform was introduced with the use of field programmable gate arrays (FPGA) ${ }^{2}$ that yielded a 95\% performance improvement (sub $5 \mu$ s end to end) compared to homogeneous software. ${ }^{3-5,7}$ The latest generation of the heterogeneous (FPGA, GPU) application suite enables new business models by realizing financial risk simulations and deep learning in real time.

\section{Description-driven systems.}

The description-driven approach to software generation was again a disruptive response to meet demands for higher quality and quicker delivery, with lower costs. LSEG Technology introduced a patented business rule engine in 1998, which allowed flexibility in specifying business features without requiring redeployment or upgrades. The core of this approach was extensible to the description of an entire system (that is, data model, business functionality, work flows, user interface (UI), and deployment).

Using a combination of theorem provers and code generators, it has been

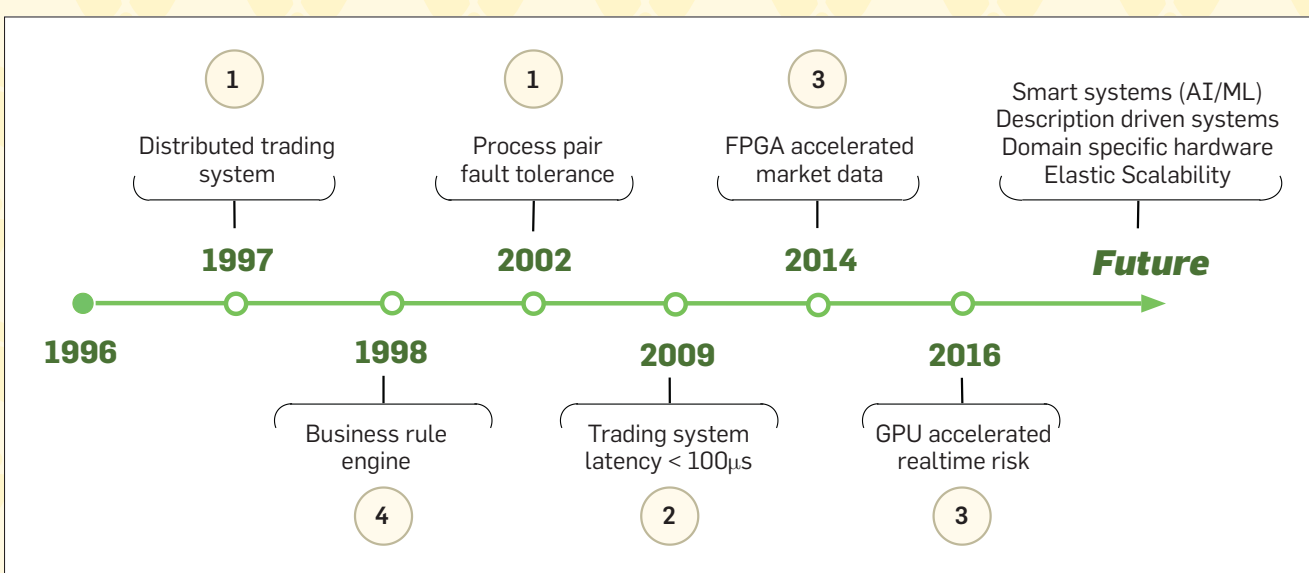

Figure 2. Themed milestones of creative disruption.

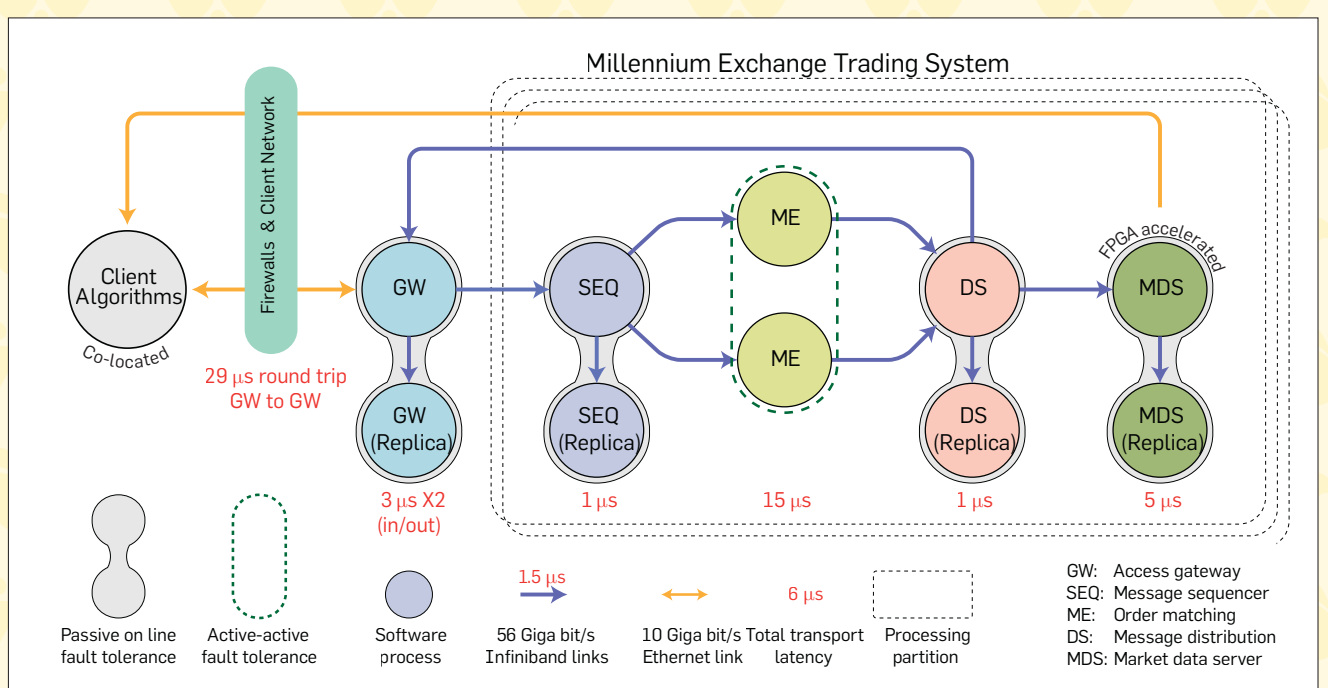

Figure 3. An abstract view of the Millennium Exchange trading system.

shown to generate approximately $80 \%-90 \%$ of the code of a system. An initial prototype of a fully functional post-trade clearing system demonstrated that a 5k-line system description will generate up to $950 \mathrm{k}$ lines of $\mathrm{C}++$, JavaScript, or SQL code.

\section{Summary}

The success of LSEG Technology bears testimony that despite contextual barriers, organizations in this region can indeed become globally competitive technology leaders in specialized niche markets. The early application of 'creative disruption' to a niche market with immense growth potential has proven to be a very effective tool and strategy.

The increasing pace of technological advancements warrant a balanced outlook toward agility, farsighted bets on technology, and investments in intellectual capital, to exploit the unfolding opportunities of the future. ${ }^{1}$

\section{References}

1. Bloomberg LP U.S., 2019; https:// bloom.bg/2KG8NG

2. Businesswire.com. 2013; http://bit. ly/2R5Brlm

3. Finextra.com. 2016; http://bit. ly/2wMtF6D

4. Fnlondon.com. 2016; http://bit ly/2K79sRC

5. Ibsintelligence.com. 2016; http://bit. ly/2WyHC7w

6. London Stock Exchange Group PLC U.K. 2019; https://www.lseg.com/ Iseg-technology

7. Thomsonreuters.com. 2015; https:// tmsnrt.rs/2wNWvUg

8. Waterstechnology.com. 2017; http:// bit.ly/31pIsSV

9. Waterstechnology.com. 2018; http:// bit.ly/2wOLvpM

Ajit Samaranayake (ajit@lseg.com) is chief scientist at LSEG Technology, Colombo, Sri Lanka.

Sampath Tilakumara (sampath@lseg. com) is head of technology at LSEG

Technology, Colombo, Sri Lanka.

Thayaparan Sripavan (thaya@lseg.com) is head of hardware-accelerated systems at LSEG Technology, Colombo, Sri Lanka. Rasika Withanawasam (rasikaw@lseg. com) is senior software architect at LSEG Technology, Colombo, Sri Lanka.

Copyright held by authors/owners 